

\title{
Early Detection of Diabetic Retinopathy Using K-means Clustering Algorithm and Ensemble Classification Approach
}

\author{
Nandeeswar Sampigehalli Basavaraju ${ }^{1 *}$ \\ ${ }^{1}$ Presidency University, Bangalore, India \\ * Corresponding author's Email: technandy1@gmail.com
}

Shanmugarathinam Ganesarathinam ${ }^{1}$

\begin{abstract}
In recent decades, the automatic recognition of diabetic retinopathy is an emerging research topic, where diabetic retinopathy is the major cause of vision loss in human beings. The early recognition of diabetic retinopathy lesions like the optic disc, cotton wools, hemorrhages, exudates, and Microaneurysms reduces the growth rate of diabetic retinopathy disease and assists clinicians in early treatment. However, the monitoring, and recognition of diabetic retinopathy lesions are tiring, cyclic and error-prone processes. To overcome these problems, a new automated diabetic retinopathy detection model is proposed in this paper. Firstly, the retinal fundus images are acquired from Kaggle retinal dataset and DiaRetDB1 dataset, and the acquired raw fundus image quality is improved using histogram equalization technique, Haar wavelet transform, discrete wavelet transform and Gaussian matched kernel filtering method. Further, the k-means clustering algorithm and Gaussian blur technique are applied for fovea and blood vessel removal, and to highlight the optic disc regions. Next, color histogram features are extracted from the segmented regions, and the obtained features are fed to an ensemble classifier for classifying the healthy, and DR subjects. The simulation results show that the proposed k-means based ensemble model achieved $93.29 \%$ and $99.37 \%$ of accuracy on the Kaggle retinal dataset and DiaRetDB1 dataset, which are better compared to the existing state of the art models.
\end{abstract}

Keywords: Color histogram features, Diabetic retinopathy, Ensemble classifier, Gaussian matched kernel filters, Histogram equalization, K-means clustering algorithm.

\section{Introduction}

Diabetic retinopathy (DR) is a retinal disease, which is the major cause of blindness in human beings. There are no symptoms that appeared for DR until it becomes severe, so earlier detection, and treatment decreases the growth rate of this disease [1$3]$. In these circumstances, multiple lesions are generated on the retinal surface like hemorrhages, cotton wools, exudates, and microaneurysms that represents the presence of DR [4]. Manual examination of DR depends on patient conditions, where the detection consumes more time, error and arduous [5-6]. Additionally, due to the lack of medical resources and a large number of diabetic patients, DR is not timely treated and diagnosed which leads to blindness and irreversible visual loss, especially in developing countries like India [7]. So, an automated screening system is developed by the researchers for an early diagnosis of DR. In the past two decades, several automated models are developed by the researchers for detecting DRrelated lesions in the color retinal fundus images. The models like region growing, hybrid method, mathematical morphology, and wavelet-based approaches focused on detecting microaneurysms [8]. On the other hand, the models like pixel classification, and mathematical morphology focused on detecting hemorrhages for early diagnosis of DR [9]. However, the aforementioned models are usually found to be defective, due to a lack of interpretability. The major contributions of this paper are listed below;

- The input retinal fundus images are collected from two benchmark datasets such as kaggle retinal dataset and the DiaRetDB1 dataset. In the application like medical image processing, it is necessary to select the proper datasets, or 
else the intended operations like segmentation, classification gets affected.

- The raw retinal fundus image quality is improved using histogram equalization technique, haar wavelet transform, discrete wavelet transform (DWT) and gaussian matched kernel filter.

- Further, a k-means clustering algorithm is employed on the denoised images to remove blood vessels, and fovea. The k-means algorithm includes the benefits like scales to large medical datasets, is simple in implementation, easily adapts, and guarantees convergence. Further, the optic disc region is highlighted by using the gaussian blur technique.

- Lastly, color histogram feature vectors are extracted from the segmented regions, and then the ensemble classification approach (decision tree, support vector machine (SVM), random forest, and Naïve Bayes) is proposed to classify the healthy, and DR subjects. The proposed kmeans based ensemble model performance is validated in terms of sensitivity, accuracy, and specificity.

This paper is pre-arranged as follows; some recent research papers belong to the topic "DR detection" are surveyed in section 2. The proposed automated DR detection model is explained in section 3. Quantitative and comparative evaluation of the proposed automated DR detection model is given in section 4 . The conclusion of the research is given in section 5 .

\section{Related works}

Kumar [10] developed a model for early diagnosis of DR by segmenting hemorrhages and microaneurysm. At first, mathematical morphology operation was utilized for pre-processing, and blood vessel segmentation. Next, watershed transformation technique was utilized to segment optic disc, and to localize fovea. Further, feature extraction was accomplished by using circularity, area, number of lesions, perimeter, minor axis length, major axis length, and aspect ratio to extract the features from the segmented region. Lastly, radial basis function neural network (RBFNN) was employed to classify healthy, and DR individuals. The presented model performance was evaluated on the DiaRetDB1 dataset in light of specificity and sensitivity. The data training was faster in RBFNN, but the classification was slower in comparison with other deep learning models because every node in the hidden layer needs to calculate RBF for classification. Jadhav [11] introduced a novel automated DR detection system by segmenting soft exudates, hard exudates, microaneurysm, and hemorrhages. At first, optic disc removal was carried out by open-close watershed transformation technique and then grey-level thresholding was applied for removing blood vessels from the fundus images. After segmentation, Shannon's entropy, local binary pattern (LBP), Kapur's entropy and texture energy measurement were used for extracting the features. The obtained features were high dimensional, so a modified gear and steering-based rider optimization algorithm were used for selecting the optimal features that were fed to deep belief network (DBN) to classify severe, moderate, earlier and normal stages. However, the class imbalance was a major issue in DBN model.

Shankar [12] applied contrast limited adaptive histogram equalization (CLAHE) technique to improve the contrast of collected retinal fundus images. Next, the histogram method and inception ResNet V2 model were used for segmentation and feature extraction. Then, the extracted features were fed to deep neural network with moth search optimization (DNN-MSO) model to classify the dissimilar stages of DR. Simulation results confirmed that the developed model attained significant performance in DR detection on messidor DR dataset. However, the massive amount of unlabeled information in DNN generates issues in pattern learning. Qummar [13] ensemble five convolution neural network (CNN) models like dense 169, resnet 50 , inception 3 , dense 121 , and xception for encoding the discriminative features, and improving the classification of healthy and DR individuals. The simulation outcomes showed that the developed model significantly detects the stages of DR related to the existing models on the kaggle dataset. Still, the developed model was computationally expensive for identifying the best hypothesis. In addition, shankar [14] presented a new automated model; hyper parameter tuning inception v4 (HPTI v4) for detecting and classifying the stages of DR. Firstly, the CLAHE approach was used for image denoising and then histogram-based segmentation method was utilized for blood vessel segmentation. Next, the HPTI v4 model was applied for extracting the discriminative feature values from segmented retinal fundus images. Finally, multilayer perceptron (MLP) classifier was used for classifying the stages of DR. The statistical analysis confirmed that the presented model obtained better performance in DR detection on the MESSIDOR dataset. In image classification, the MLP classifier includes two major concerns such as overfitting and vanishing gradient. 
Gadekallu [15] introduced a new automated system for early detection of DR in the retinal fundus images. Initially, the acquired raw images were normalized using the standard scalar approach and then the rich feature values were extracted by applying principal component analysis. Further, feature dimensionality reduction was accomplished by the firefly algorithm, and the obtained optimal feature values were fed to DNN for classification. Statistical analysis shows the superiority of the developed model in DR detection by means of recall, accuracy, and precision. Romero-Oraá [16] enhanced the retinal structures, and normalize the image appears to achieve effective performance in DR detection. Secondly, manual segmentation was accomplished to locate retinal backgrounds, optic disc, fovea, and vasculatures. Additionally, feature vectors were extracted from the segmented regions and then feature selection was accomplished by using a fast correlation-based filter to select the optimal feature vectors. Finally, classification was carried out by the MLP classifier to classify true lesions from the residual candidates. However, the inclusion of lowlevel features in the high-level layers of DNN and MLP classifiers leads to the poor classification in the large datasets.

Pundikal and Holi [17] developed a new model for detecting microaneurysms from the retinal fundus images for early diagnosis of DR. Firstly, the images were acquired from DiaretDB1 and e-ophtha dataset. Further, normalization, gradient weighting, low light image enhancement, and shade correction were used to improve the quality of collected images. Next, the otsu thresholding and hessian based filter were used to extract the foreground object like microaneurysms from the pre-processed retinal fundus images. Lastly, grey wolf optimization (GWO) algorithm was used for predicting the correctness of microaneurysms candidates. In microaneurysms segmentation, GWO algorithm includes three major concerns such as low solving precision, bad local searching capability, and slow convergence.

Qomariah [18] presented a new deep learning model that modifies UNet by utilizing residual units with the modified identity mapping for segmenting the microaneurysms. The developed model significantly enriches the features for overcoming the feature loss, and the mean weighted loss function in the developed model solves the issue of pixel imbalance between the background and the microaneurysms. Simulation analysis showed that the developed model obtained better performance in microaneurysms detection in light of sensitivity. However, overfitting was a major issue in UNet model for microaneurysms detection.
Putra [19] used contrast limited adaptive histogram equalization, homomorphic and morphology contrast enhancement techniques for improving the quality of collected images. The preprocessed images were fed to feature extraction layer of ResNet101, ResNet50, GoogLeNet, and ResNet18. The dimension of the extracted features was reduced by utilizing relief and principle component analysis techniques. Lastly, the SVM-Naïve Bayes was applied for replacing the fully connected layer in convolutional neural network to optimize and to speed up the classification process. However, the convolutional neural network model was computationally expensive where it needs higher end graphics processing units' system to process large amount of data. To address the above stated issues, a novel k-means based ensemble model is proposed in this research article for early diagnosis of DR in the retinal fundus images.

\section{Methodology}

The proposed automated DR detection model majorly includes five steps like data collection: Kaggle retinal dataset and DiaRetDB1 dataset, data pre-processing: histogram equalization, haar wavelet transform, DWT and gaussian matched kernel filter, segmentation: k-means clustering algorithm and gaussian blur technique, feature extraction: color histogram, and classification: ensemble classifier.

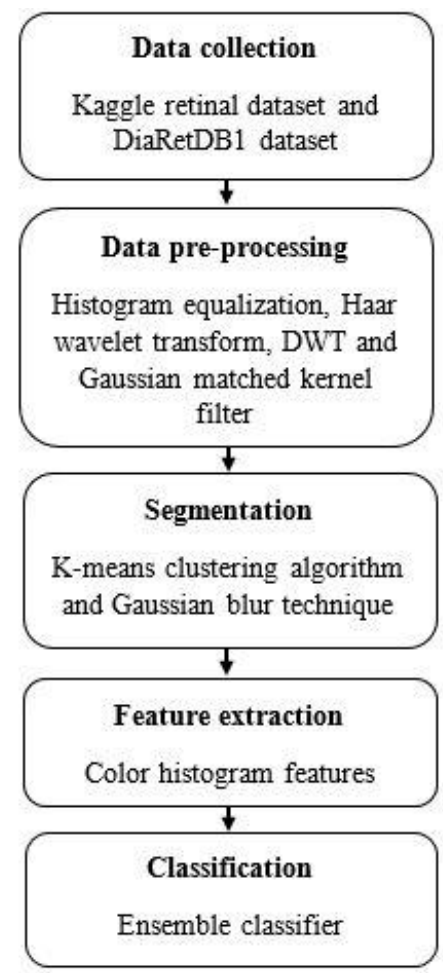

Figure. 1 Workflow of proposed k-means based ensemble model 
The workflow of the proposed k-means based ensemble model is graphically denoted in Fig. 1.

\subsection{Data collection}

In this paper, the proposed DR detection model performance is validated on kaggle retinal dataset and DiaRetDB1 dataset. The kaggle retinal dataset consists of 88702 retinal fundus images in that 17740 fundus images are used for testing, and 70962 fundus images are utilized for training. In kaggle retinal dataset, every individual includes two fundus images with different pixel values ranging from $433 \times 289$ to $5184 \times 3456$ pixels. Based on the early treatment DR study scale, the expert validated every fundus

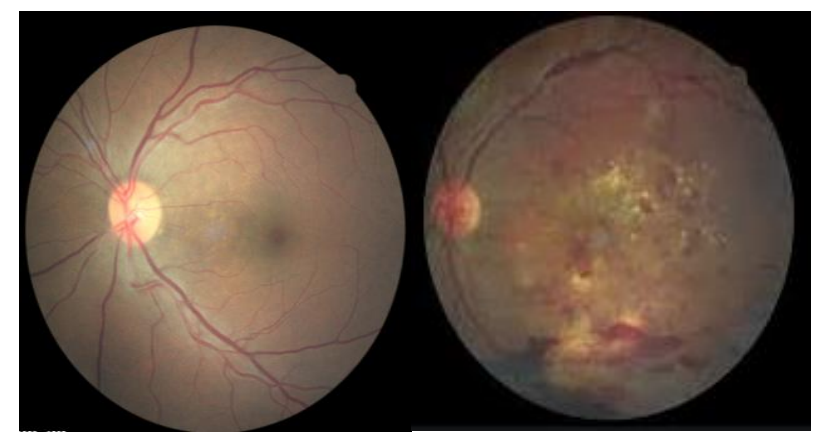

Figure. 2 Sample images of kaggle retinal dataset
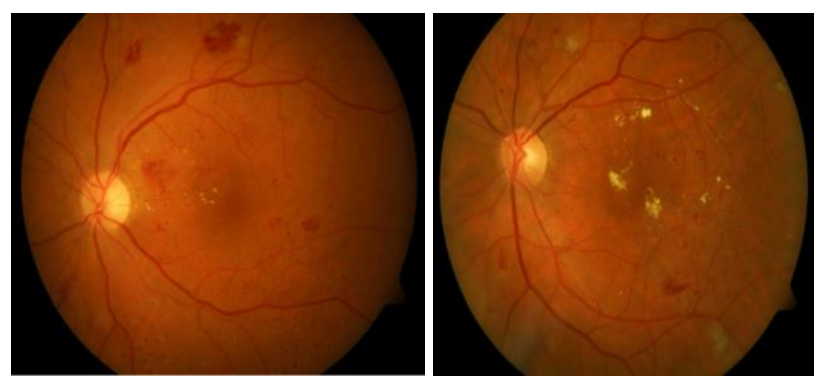

Figure. 3 Sample images of DiaRetDB1 dataset

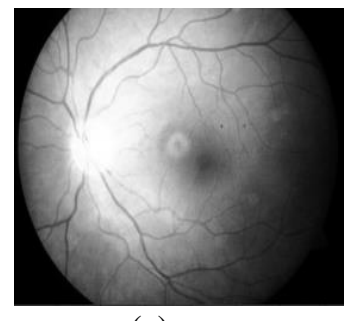

(a)

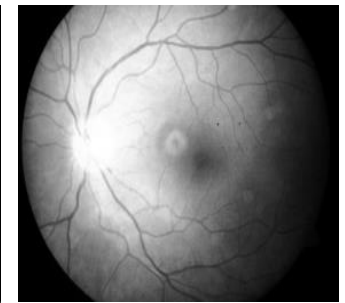

(b)

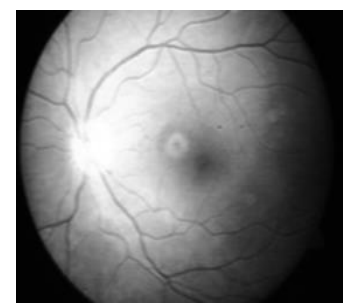

(c)

Figure. 4 Denoised fundus images: (a) grayscale image, (b) transformed image, and (c) gaussian matched kernel filter image image for the presence of DR with a scale of 0-4 [20]. The sample images of the kaggle retinal dataset are denoted in Fig. 2.

Dataset link: https://www.kaggle.com/c/diabeticretinopathy-detection/data

The DiaRetDB1 dataset comprises 89 fundus images with a pixel size of $1500 \times 1152$ in that 5 fundus images belong to normal individuals, and the remaining 84 fundus images belong to the subjects affected from microaneurysms (mild nonproliferative sign). In this dataset, the fundus images are recorded using 50o field of view camera with different image settings [21]. Sample images of the DiaRetDB1 dataset is indicated in Fig. 3.

Dataset

link:

https://www.it.lut.fi/project/imageret/diaretdb1/

\subsection{Data pre-processing}

After retinal fundus image collection, the collected images are converted into grayscale images, and then pre-processing is performed by histogram equalization technique, haar wavelet transform, DWT and gaussian matched kernel filter. Histogram equalization technique equally transforms image histogram values, which results in contrast enhancement [22]. The general formula of the histogram equalization technique is represented in Eq. (1).

$$
\begin{aligned}
& p_{I}=\frac{\text { number of pixels with intensity } n}{\text { Total number of pixels }} \\
& n=0,1 \ldots, L-1
\end{aligned}
$$

where $p$ indicates normalized histogram of an image $I, L$ represents some possible intensity values, which are often 256. Then, haar wavelet transform, and DWT are applied on normalized image $p_{I}$ for reconstructing the image with no loss of information, which helps in effective noise removal. In addition to this, gaussian matched kernel filter is employed to enhance the quality of vessel-like structures in the fundus images. The motivation behind gaussian matched kernel filtering method is to approximate the shape of vessel-like structures by utilizing a gaussian template [23]. Gaussian template $G(x, y)$ is mathematically indicated in Eq. (2).

$$
G(x, y)=-\exp \left(-\frac{x^{2}+y^{2}}{2 \sigma^{2}}\right),|y| \leq \frac{u}{2}
$$

where, $x$ and $y$ represents the rows and columns of fundus image, $\sigma$ represents the average width of vessel-like structures and $u$ states pixel length of 
vessel-like structures. The denoised fundus images are graphically represented in Fig. 4.

\subsection{Segmentation}

After image enhancement, a k-means clustering algorithm is used for removing blood vessels, and fovea. In k-means clustering, the enhanced fundus images are partitioned into a $k$ number of groups or $k$ number of disjoint clusters. In segmentation, the kmeans clustering algorithm comprises two major phases (i) computes $k$ centroids, and (ii) determines the clusters, which have the nearest centroid with respect to the data points [24]. In this research study, euclidean distance measure is applied to determine the distance of the nearest centroid. In the k-means clustering algorithm, every cluster is defined by its centroid, and its member objects. The centroid for every cluster is the point to which the distance from all the objects in the cluster is reduced. The step by step process of the k-means clustering algorithm is given as follows;

- A number of clusters $k$ and centers are initialized.

- Determine the euclidean distance $d$ between the center $c_{k}$ and image pixel using Eq. (3).

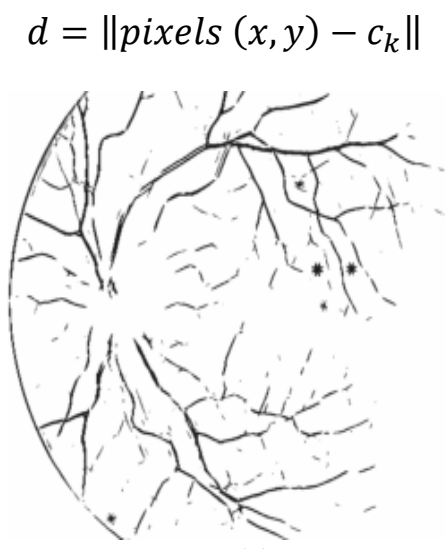

(a)

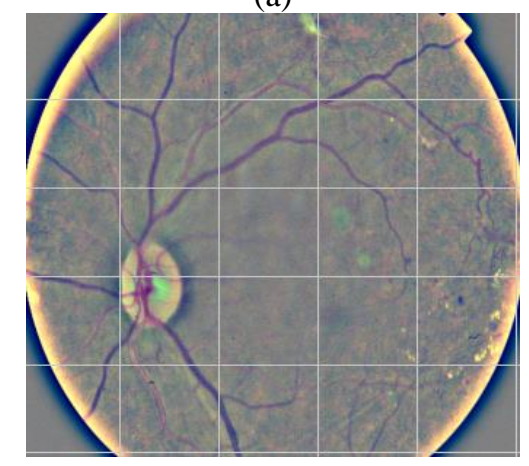

(b)

Figure. 5 Segmented images: (a) output of k-means clustering algorithm and (b) output of gaussian blur technique
- Allocate all the pixel values to the nearest center based on euclidean distance $d$.

- Re-calculate the new position of the center by using Eq. (4), after all the pixel values are allocated.

$$
c_{k}=\frac{1}{k} \sum_{y \in c_{k}} \sum_{x \in c_{k}} \text { pixels }(x, y)
$$

- The aforementioned steps are repeated until the error or tolerance value is satisfied.

- Finally, reshape the cluster pixels into a medical image.

Further, the gaussian blur technique is applied to the output image of the k-means clustering algorithm to improve the visual effect of the image by utilizing a gaussian function. The gaussian smoothing is also used as a pre-processing technique in medical image processing applications to improve the image structures at different scales. In this research, the gaussian blur technique is used for enhancing or highlighting the optic disc regions that help in achieving better classification performance. The segmented images are graphically represented in Fig. 5 .

\subsection{Feature extraction and classification}

After image segmentation, color histogram features are extracted from the segmented regions for better classification of DR stages. The color histogram is the extensively used method to extract the color features of an image. The color histogram feature vectors denote the fundus image from a different perspective and also it states the frequency distribution of color bins in a fundus image. The color histogram method counts the similar pixel and extracts the discriminative feature vectors. The extracted feature vectors are given as the input to the ensemble classifier to classify the different stages of DR. In this paper, the ensemble classifier combines four machine learning classifiers like SVM, decision tree, naïve bayes and random forest to classify DR stages. The ensemble classifier improves the machine learning results by combining numerous models, which results in better classification performance compared to individual models. The motivation behind ensemble classifiers is to learn a set of classifiers and vote for the best results (higher classification accuracy). The ensemble classifiers make better classification and achieve significant performance than single contributing model. Hence, the ensemble classifiers diminish the dispersion or spread of the model performance. The description of 
four machine learning classifiers like SVM, decision tree, naïve bayes and random forest are given below;

SVM: It is a supervised classification technique, which is used for both classification and regression purposes. Commonly, an SVM classifier is used for classification problems, where it uses a hyperplane that effectively divides the dataset into binary classes [25].

Random forest: It is an ensemble learning technique applied for regression and classification by constructing a multitude of decision trees at the training stage and outputting the classes, which is the average prediction of the individual or single tree [26].

Decision tree: It is a tree-like structure classification technique, where its branches state decision rules, internal nodes indicate dataset features, and leaf node denotes output [27]. In this classifier, a decision is carried out based on the features of the given dataset.

Naïve bayes: It is a probabilistic classifier, which works based on the bayes theorem with a strong independence assumption between the data features [28]. Naïve bayes classifier is highly scalable, but it needs more variables or features to solve learning problems. Experimental analysis of the proposed kmeans based ensemble model is given in section 4 .

\section{Simulation results}

In DR detection, the proposed k-means based ensemble model performance is simulated by Python 3.7.3 environment on a computer with 8 GB RAM, Intel Core i5 processor, and Windows 10 (64 bit) operating system. In this article, the efficiency of the proposed $\mathrm{k}$-means based ensemble model is analysed by comparing with a few benchmark models such as watershed transform with RBFNN model [10], improved rider optimization algorithm enabled with deep learning [11], deep learning ensemble approach [13], and decomposed MLP classifier [16] on DiaRetDB1, and kaggle retinal datasets. In this scenario, k-means based ensemble model performance is analysed by means of accuracy, specificity, and sensitivity. In medical image classification, accuracy is determined as the ratio of correctly predicted observations to the entire observations. Further, sensitivity computes the number of true positives, and specificity finds the number of true negatives that are precisely recognized. The mathematical representation of accuracy, specificity and sensitivity is denoted in the Eq. (5-7).

$$
\text { Accuracy }=\frac{T P+T N}{T N+T P+F N+F P} \times 100
$$

$$
\begin{aligned}
& \text { Specificity }=\frac{T N}{T N+F P} \times 100 \\
& \text { Sensitivity }=\frac{T P}{T P+F N} \times 100
\end{aligned}
$$

where, $T P$ indicates true positive, $F P$ states false positive, $T N$ denotes true negative, and $F N$ states false negative.

\subsection{Analysis of DiaRetDB1 dataset}

In this segment, the proposed k-means based ensemble model performance is validated on the DiaRetDB1 dataset, which comprises 89 color retinal fundus images. In this experiment, $80 \%$ of retinal fundus images are applied for model training, and the remaining $20 \%$ of the retinal fundus images are applied for model testing. By investigating Table 1, the performance analysis is accomplished with individual classifiers (SVM, decision tree, naïve bayes and random forest) and ensemble classifiers using accuracy, specificity, and sensitivity. From Table 1, the ensemble classifier obtained maximum accuracy value of $99.38 \%$, specificity value of $96.3 \%$, and sensitivity value of $97.39 \%$ in DR detection on DiaRetDB1 dataset. Sample classified images of DiaRetDB1 dataset is indicated in Fig. 6, and the graphical representation of k-means based ensemble model performance on DiaRetDB1 dataset is denoted in Fig. 7.

\subsection{Analysis of kaggle retinal dataset}

In this segment, the proposed k-means based ensemble model performance is validated on kaggle retinal dataset which consists of 88702 color retinal fundus images in that $80 \%$ (70962) of the fundus images are used for model training, and $20 \%$ (17740) of the fundus images are utilized for model testing. By inspecting Table 2, the ensemble classifier obtained a maximum accuracy value of $93.29 \%$, specificity value of $85.8 \%$ and sensitivity value of $84.98 \%$ in DR detection, where the obtained values are better related to the comparative classifiers such as SVM, decision tree, random forest, and naïve bayes. Sample classified images of the kaggle retinal dataset is denoted in Fig. 8. The graphical representation of the proposed k-means based ensemble model performance on the kaggle retinal dataset is represented in Fig. 9. 
Table 1. Performance analysis of k-means based ensemble model performance on DiaRetDB1 dataset

\begin{tabular}{|c|c|c|c|}
\hline Classifiers & Accuracy (\%) & Sensitivity (\%) & Specificity (\%) \\
\hline SVM & 98.42 & 96 & 92 \\
\hline Decision tree & 96.21 & 94 & 93.2 \\
\hline Naïve Bayes & 95.78 & 94.25 & 91.5 \\
\hline Random forest & 99.34 & 97.2 & 91.32 \\
\hline Ensemble classifier & $\mathbf{9 9 . 3 8}$ & $\mathbf{9 7 . 3 9}$ & $\mathbf{9 6 . 3 0}$ \\
\hline
\end{tabular}

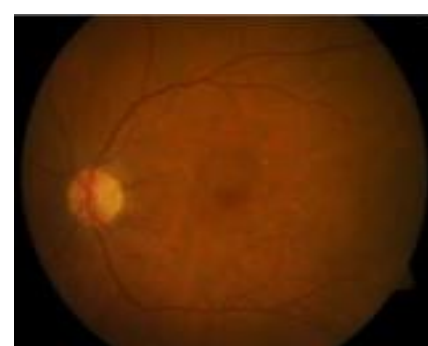

(a)

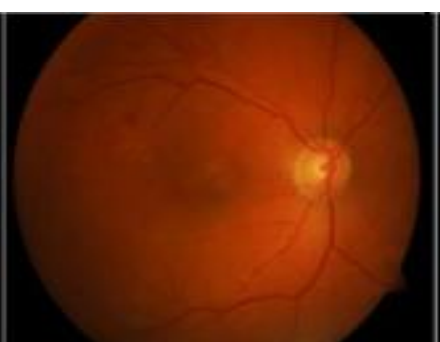

(b)

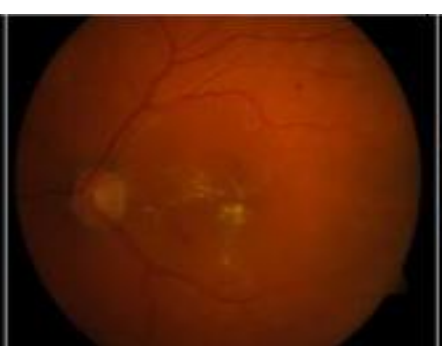

(c)

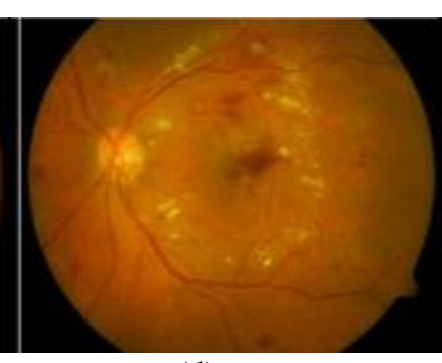

(d)

Figure. 6 Sample classified images of DiaRetDB1 dataset: (a) Normal, (b) earlier, (c) moderate, and (d) severe

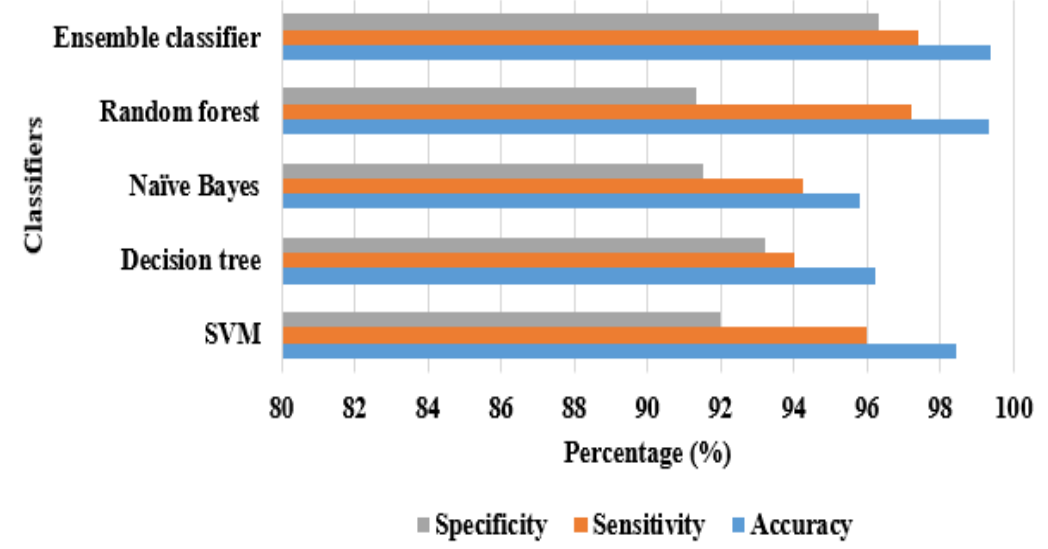

Figure. 7 Graphical illustration of k-means based ensemble model performance on DiaRetDB1 dataset

Table 2. Performance analysis of k-means based ensemble model performance on kaggle retinal dataset

\begin{tabular}{|c|c|c|c|}
\hline Classifiers & Accuracy (\%) & Sensitivity (\%) & Specificity (\%) \\
\hline SVM & 93 & 82.02 & 81.02 \\
\hline Decision tree & 88 & 82 & 81.87 \\
\hline Naïve Bayes & 87 & 84.09 & 84 \\
\hline Random forest & 87 & 82 & 81.12 \\
\hline Ensemble classifier & $\mathbf{9 3 . 2 9}$ & $\mathbf{8 4 . 9 8}$ & $\mathbf{8 5 . 8 2}$ \\
\hline
\end{tabular}



(a) (b)

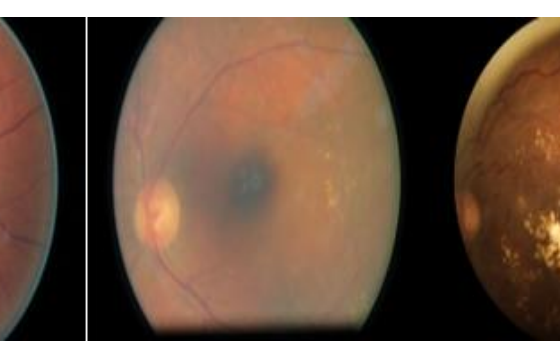

(c)



(d) (e)

Figure. 8 Sample classified images of kaggle retinal dataset: (a) normal, (b) mild, (c) moderate, (d) severe, and (e) proliferative DR 


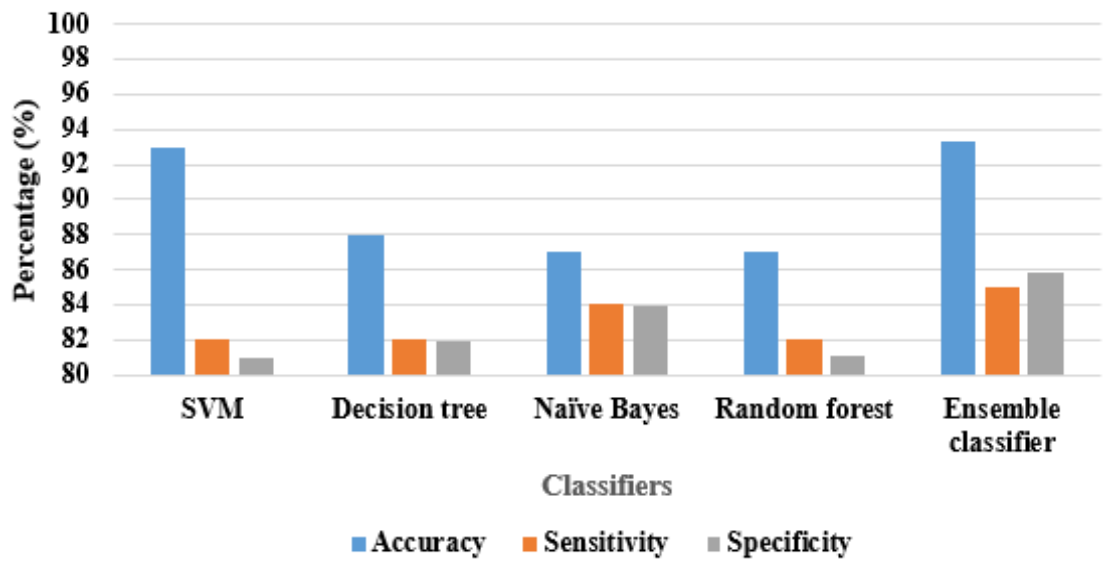

Figure. 9 Graphical illustration of k-means based ensemble model performance on kaggle retinal dataset

Table 3. Comparative analysis between the proposed k-means based ensemble model and the existing models

\begin{tabular}{|c|c|c|c|c|}
\hline Models & Dataset & Accuracy (\%) & Sensitivity (\%) & Specificity (\%) \\
\hline Watershed transform with RBFNN [10] & DiaRetDB1 & - & 87 & 93 \\
\hline $\begin{array}{c}\text { Improved rider optimization algorithm } \\
\text { enabled with deep learning [11] }\end{array}$ & DiaRetDB1 & 93.18 & 86.34 & 95.45 \\
\hline Deep learning ensemble approach [13] & Kaggle retinal & 80.8 & 51.5 & 86.72 \\
\hline Decomposed MLP [16] & DiaRetDB1 & 91.80 & 95 & 90.24 \\
\hline K-means based ensemble model & DiaRetDB1 & 99.38 & 97.39 & 96.30 \\
\cline { 2 - 5 } & Kaggle retinal & 93.29 & 84.98 & 85.82 \\
\hline
\end{tabular}

\subsection{Comparative analysis}

The comparative analysis between the proposed k-means based ensemble model and the existing models is denoted in table 3. Kumar [10] applied mathematical morphology operation for image preprocessing and for segmenting hemorrhages, microaneurysm and blood vessels from the denoised fundus images. In addition, the watershed transformation technique was applied to segment optic disc and fovea regions. Further, minor axis length, circularity, aspect ratio, major axis length, area, number of lesions and perimeter were used for feature extraction. Lastly, the RBFNN model was used for classifying normal, earlier, moderate and severe individuals. The simulation result showed that the presented model obtained $87 \%$ of sensitivity and $93 \%$ of specificity on the DiaRetDB1 dataset. Additionally, jadhav [11] used the open-close watershed transformation technique and grey level thresholding for optic disc and blood vessel removal. Further, feature extraction and selection were carried out by using Shannon's entropy, LBP, Kapur's entropy, texture energy measurement, and modified gear and steering-based rider optimization algorithm. The obtained optimal features were given as the input to the DBN model for classifying severe, moderate, earlier, and normal stages. The simulation results showed that the developed model obtained $93.18 \%$ of accuracy, $86.34 \%$ of sensitivity, and $95.45 \%$ of specificity on the DiaRetDB1 dataset.
Qummar [13] ensemble five CNN models; dense169, Resnet50, Inceptionv3, dense121, and xception to classify normal, mild, moderate, severe and proliferative DR stages. The experimental results showed that the developed model obtained $80.80 \%$ of accuracy, $51.50 \%$ of sensitivity, and $86.72 \%$ of specificity on the kaggle retinal dataset. RomeroOraá [16] accomplished manual segmentation to detect optic disc, retinal backgrounds, fovea and vasculatures. Further, 100 feature sub-sets were extracted from the segmented regions and then a fast correlation-based filter was used for feature selection. The selected feature sub-sets were fed to the MLP classifier to classify true lesions from the residual candidates. The simulation result showed that the presented model obtained $95 \%$ of sensitivity, $91.80 \%$ of accuracy and $90.24 \%$ of specificity on DiaRetDB1 dataset. Compared to these existing models, the proposed $\mathrm{k}$-means based ensemble model obtained better performance in DR detection on DiaRetDB1 and kaggle retinal datasets. On the large medical datasets, the ensemble models help in improving the machine learning results by combining multiple models, as denoted in the section 3.4. The proposed ensemble model delivers significant classification results compared to the individual machine learning models, as seen in the Tables 1 and 2. The strength of ensemble models includes; reduction of variance to avoid over-fitting concern, robust performance on the outliers, accommodates high non-linear interactions, requires only little 
additional parameter tuning, and computationally effective. As seen in the quantitative analysis section, the K-means based ensemble model effectively overcomes the concerns mentioned in the literature papers [13-16, 18, 19].

\section{Conclusion}

In this research paper, a new k-means based ensemble model is proposed for improving the performance of DR detection in the color retinal fundus images. The proposed $\mathrm{k}$-means based ensemble model includes two major steps as segmentation and classification. In this article, a kmeans clustering algorithm is employed to eliminate fovea, and blood vessel regions from the enhanced retinal fundus images, and then the optic disc region is highlighted by applying gaussian blur technique. Further, the color histogram features are fed to an ensemble machine learning classifier to classify healthy and DR subjects. Compared to the prior deep learning classification methods, the computational complexity of the proposed ensemble classifier is linear or limited. The experimental analysis showed that the proposed $\mathrm{k}$ means-based ensemble model obtained significant performance in DR detection in light of sensitivity, accuracy and specificity. As seen in the comparative analysis section, the proposed kmeans based ensemble model obtained $93.29 \%$ of classification accuracy on kaggle retinal dataset, and $99.37 \%$ of accuracy on DiaRetDB1 dataset, which are effects related to the comparative models. In future work, global level feature descriptors are included in the k-means based ensemble model to further enhance DR detection performance.

\section{Conflicts of interest}

The authors declare no conflict of interest.

\section{Author contributions}

The paper background work, conceptualization, methodology, dataset collection, implementation, result analysis and comparison, preparing and editing draft, visualization have been done by first author. The supervision, review of work and project administration, have been done by second author.

\section{References}

[1] Q. Xie, Y. Liu, H. Huang, B. Hong, J. Wang, H. Han, and Y. Liu, "An innovative method for screening and evaluating the degree of diabetic retinopathy and drug treatment based on artificial intelligence algorithms", Pharmacological Research, Vol. 159, pp. 104986, 2020.
[2] J. Krause, V. Gulshan, E. Rahimy, P. Karth, K. Widner, G. S. Corrado, L. Peng, and D. R. Webster, "Grader variability and the importance of reference standards for evaluating machine learning models for diabetic retinopathy", Ophthalmology, Vol. 125, No. 8, pp. 1264-1272, 2018.

[3] S. Wan, Y. Liang, and Y. Zhang, "Deep convolutional neural networks for diabetic retinopathy detection by image classification", Computers \& Electrical Engineering, Vol. 72, pp. 274-282, 2018.

[4] T. Shanthi, and R. S. Sabeenian, "Modified Alexnet architecture for classification of diabetic retinopathy images", Computers \& Electrical Engineering, Vol. 76, pp. 56-64, 2019.

[5] Z. Gao, J. Li, J. Guo, Y. Chen, Z. Yi, and J. Zhong, "Diagnosis of diabetic retinopathy using deep neural networks", IEEE Access, Vol. 7, pp. 33603370, 2018.

[6] K. Xu, D. Feng, and H. Mi, "Deep convolutional neural network-based early automated detection of diabetic retinopathy using fundus image", Molecules, Vol. 22, No. 12, pp. 2054, 2017.

[7] W. Zhang, J. Zhong, S. Yang, Z. Gao, J. Hu, Y. Chen, and Z. Yi, "Automated identification and grading system of diabetic retinopathy using deep neural networks", Knowledge-Based Systems, Vol. 175, pp. 12-25, 2019.

[8] G. T. Zago, R. V. Andreão, B. Dorizzi, and E. O. T. Salles, "Diabetic retinopathy detection using red lesion localization and convolutional neural networks", Computers in Biology and Medicine, Vol. 116, pp. 103537, 2020.

[9] T. Li, Y. Gao, K. Wang, S. Guo, H. Liu, and H. Kang, "Diagnostic assessment of deep learning algorithms for diabetic retinopathy screening", Information Sciences, Vol. 501, pp. 511-522, 2019.

[10] S. Kumar, A. Adarsh, B. Kumar, and A. K. Singh, "An automated early diabetic retinopathy detection through improved blood vessel and optic disc segmentation", Optics \& Laser Technology, Vol. 121, pp. 105815, 2020.

[11] A. S. Jadhav, P. B. Patil, and S. Biradar, "Optimal feature selection-based diabetic retinopathy detection using improved rider optimization algorithm enabled with deep learning", Evolutionary Intelligence, pp. 1-18, 2020.

[12] K. Shankar, E. Perumal, and R. M. Vidhyavathi, "Deep neural network with moth search optimization algorithm based detection and classification of diabetic retinopathy images", $S N$ Applied Sciences, Vol. 2, No. 4, pp. 1-10, 2020. 
[13] S. Qummar, F. G. Khan, S. Shah, A. Khan, S. Shamshirband, Z. U. Rehman, I. A. Khan, and W. Jadoon, "A deep learning ensemble approach for diabetic retinopathy detection", IEEE Access, Vol. 7, pp. 150530-150539, 2019.

[14] K. Shankar, Y. Zhang, Y. Liu, L. Wu, and C. H. Chen, "Hyperparameter tuning deep learning for diabetic retinopathy fundus image classification", IEEE Access, Vol. 8, pp. 118164-118173, 2020.

[15] T. R. Gadekallu, N. Khare, S. Bhattacharya, S. Singh, P. K. R. Maddikunta, I. H. Ra, and M. Alazab, "Early detection of diabetic retinopathy using PCA-firefly based deep learning model", Electronics, Vol. 9, No. 2, pp. 274, 2020.

[16] R. R. Oraá, M. García, J. O. Pérez, M. I. L. Gálvez, and R. Hornero, "Effective Fundus Image Decomposition for the Detection of Red Lesions and Hard Exudates to Aid in the Diagnosis of Diabetic Retinopathy", Sensors, Vol. 20, No. 22, pp. 6549, 2020.

[17] M. Pundikal, and M. S. Holi, "Detection of Microaneurysms Using Grey Wolf Optimization for Early Diagnosis of Diabetic Retinopathy", International Journal of Intelligent Engineering and Systems, Vol. 13, No. 6, pp. 208-218, 2020.

[18] D. U. N. Qomariah, H. Tjandrasa, and C. Fatichah, "Segmentation of Microaneurysms for Early Detection of Diabetic Retinopathy Using MResUNet", International Journal of Intelligent Engineering and Systems, Vol. 14, No. 3, pp. 359-373, 2021.

[19] R. E. Putra, H. Tjandrasa, and N. Suciati, "Severity classification of non-proliferative diabetic retinopathy using convolutional support vector machine", International Journal of Intelligent Engineering and Systems, Vol. 13, No. 4, pp. 156-170, 2020.

[20] C. Lam, D. Yi, M. Guo, and T. Lindsey, "Automated detection of diabetic retinopathy using deep learning", AMIA Summits on Translational Science Proceedings, pp. 147, 2018.

[21] R. V. J. P. H. Kälviäinen, and H. Uusitalo, "DIARETDB1 diabetic retinopathy database and evaluation protocol", Medical Image Understanding and Analysis, Citeseer, pp. 61, 2007.

[22] M. A. A. Wadud, M. H. Kabir, M. A. A. Dewan, and O. Chae, "A dynamic histogram equalization for image contrast enhancement", IEEE Transactions on Consumer Electronics, Vol. 53, No. 2, pp. 593-600, 2007.
[23] I. C. Aceves, F. C. Sanchez, and M. S. A. Garcia, "A novel multiscale Gaussian-matched filter using neural networks for the segmentation of Xray coronary angiograms", Journal of Healthcare Engineering, Vol. 2018, 2018.

[24] N. Dhanachandra, K. Manglem, and Y. J. Chanu, "Image segmentation using K-means clustering algorithm and subtractive clustering algorithm", Procedia Computer Science, Vol. 54, pp. 764771, 2015.

[25] D. A. Pisner, and D. M. Schnyer, "Support vector machine", Machine Learning, Academic Press, pp. 101-121, 2020.

[26] A. T. Azar, H. I. Elshazly, A. E. Hassanien, and A. M. Elkorany, "A random forest classifier for lymph diseases", Computer Methods and Programs in Biomedicine, Vol. 113, No. 2, pp. 465-473, 2014.

[27] S. Lu and S. L. Braunstein, "Quantum decision tree classifier", Quantum Information Processing, Vol. 13, No. 3, pp. 757-770, 2014.

[28] X. Zhou, S. Wang, W. Xu, G. Ji, P. Phillips, P. Sun, and Y. Zhang, "Detection of pathological brain in MRI scanning based on wavelet-entropy and naive Bayes classifier", In: Proc. of International Conference on Bioinformatics and Biomedical Engineering, pp. 201-209, 2015. 